This is a postprint version of the following published document:

A. Moreno-Barrado, M. Castro, J. Muñoz-García, and R. Cuerno (2015). Stress vs sputtering effects in the propagation of surface ripples produced by ion-beam sputtering", in Nuclear Instruments and Methods in Physics Research Section B: Beam Interactions with Materials and Atoms 365, Part A (2015) 15 December. pp. 13-16. Available in http://dx.doi.org/10.1016/j.nimb.2015.07.032

(C) Elsevier, 2015

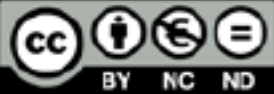

This work is licensed under a Creative Commons Attribution-NonCommercialNoDerivatives 4.0 International License. 


\title{
Stress vs sputtering effects in the propagation of surface ripples produced by ion-beam sputtering
}

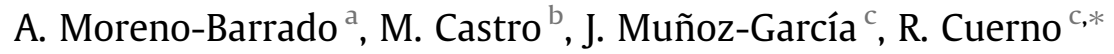 \\ a Instituto de Investigación Tecnológica (IIT) and Grupo Interdisciplinar de Sistemas Complejos (GISC), Universidad Pontificia Comillas, E-28015 Madrid, Spain \\ ${ }^{\mathrm{b}}$ GISC and Grupo de Dinámica No Lineal (DNL), Escuela Técnica Superior de Ingeniería (ICAI), Universidad Pontificia Comillas, E-28015 Madrid, Spain \\ ${ }^{\mathrm{c}}$ Departamento de Matemáticas and GISC, Universidad Carlos III de Madrid, Avenida de la Universidad 30, E-28911 Leganés, Spain
}

\begin{abstract}
Under low energy ion irradiation, periodic features (ripples) can develop on the surfaces of semiconductor materials, with typical sizes in the nanometric range. Recently, a theory of pattern formation has been able to account for the variability with the ion/target combination of the critical angle value separating conditions on ion incidence that induce the presence or the absence of ripples. Such a theory is based in the accumulation of stress in the damaged irradiated layer and its relaxation via surface-confined viscous flow. Here we explore the role of stress, and its competition with purely erosive mechanisms, to deter-mine the sign of the velocity with which the ripple pattern moves across the target plane. Based on this theory, we discuss different situations and make specific testable predictions for the change of sign in that velocity.
\end{abstract}

Keywords: Surface nanostructuring, Ion-beam sputtering, Continuum models, Stress-induced viscous flow, Ripple velocity.

\section{Introduction}

Fully-controlled surface nanopatterning by ion-beam irradiation has been an elusive goal, specially in monoelemental semiconductor materials like Silicon [1] or Germanium. The fact that recrystallization is almost negligible at room temperature makes the accumulation of defects (and, consequently, of stress) a major actor in the evolution of the damaged material. This important role for stress has been recently implemented into a continuum model for the evolution of the surface [2], which for Si irradiation has received experimental support with respect to e.g. the ion-energy dependence of the typical sizes of the patterns appearing $[3,4]$. From a physical viewpoint, stress generation coexists with sputtering in tailoring the resulting process. The former seems to be more relevant for ion-incidence angles close to the threshold angle for ripple formation [4]; the latter becomes relatively more important as the width of the amorphous layer (and hence the accumulated stress) is reduced, namely, at grazing incidence [5].

Experimentally, for the important case of Si targets, several results are consistent with the predictions of the theory in [2], namely, the existence of a critical value $\theta_{c}$ of the ion-incidence angle $\theta$, below which the pattern cannot appear $[6,7,3,8]$, the divergence of the pattern wavelength when $\theta$ approaches $\theta_{c}$ [6,7], or the

\footnotetext{
* Corresponding author.

E-mail address: cuerno@math.uc3m.es (R. Cuerno).
}

scaling of the wavelength with ion energy [3]; see further references in $[1,8]$. However, to date only a few experiments have focused on the velocity characterizing the in-plane motion of the ripple pattern. We can highlight the work by Alkemade in glass [9] or, more recently, the work by Hofsäss and collaborators on Si [10].

Interestingly, one of the predictions of the ion-induced solid flow theory is that different states of stress involve different scenarios for pattern formation [4]. Namely, depending on the relative values of stress at the free and amorphous-crystalline $(a-c)$ interfaces, the pattern formation process can occur in such a way that either: (i) Ripples form only for $\theta>\theta_{c}$ as for the experiments mentioned on $\mathrm{Si}$; since this behavior cannot be predicted by the classical Bradley-Harper (BH) theory [11], this is termed a non-BH type scenario. (ii) Patterns form for all angles which are smaller than a different critical value $\theta_{c}^{*}$, and in particular at normal incidence. Experimentally, pattern (dot) formation at $\theta=0$ does occur under impurity co-deposition or for irradiation of compound targets, see e.g. $[1,12,13]$. Indeed, the classic BH theory allowed for pattern formation (so-called parallel mode ripples) for any value of $\theta$ from normal incidence up to a critical value, beyond which parallel mode ripples disappear. Hence, we term this as a BH-type scenario.

In Ref. [4] the solid flow model has been formulated in the light of Molecular Dynamics (MD) simulations of stress generation, and contrasted with experiments on $\mathrm{Si}$. The focus was to describe the onset of ripple formation at $\theta=\theta_{c}$ through the properties of the 
ripple wavelength, in order to account for the variation of $\theta_{c}$ with the ion/target combination. The values of $\theta$ explored are sufficiently non-glancing that so-called perpendicular mode ripples do not form, see [1]. Here, we present and discuss the implications of the ensuing solid flow theory for the velocity of the ripples. The remainder of the paper is organized as follows: In Section 2 we review the main ingredients of the theory and derive the ripple velocity. In Section 3 we present the main results and discuss their experimental implications. Finally, in Section 4 we conclude and point to future developments.

\section{Continuum approach}

We next review briefly the continuum model of viscous flow driven by ion-induced stress, see further details in Refs. [2-4]. A damaged amorphous layer is assumed to have formed, with stationary mechanical properties (in our experimental context [1] this happens prior to pattern formation, after a few seconds of irradiation). As suggested by MD simulations, the amorphous layer relaxes as Newtonian fluid with a high viscosity $\eta$, ultimately due to the ion impacts [4]. Under incompressible flow, $\nabla \cdot \mathbf{v}=0$, one has

$\partial_{x} u+\partial_{z} w=0$,

$-\partial_{x} p+\eta\left(2 \partial_{x x}^{2} u+\partial_{z z}^{2} u+\partial_{x z}^{2} w\right)+\partial_{z \prime} \tau_{z z} \sin \theta=0$,

$-\partial_{z} p+\eta\left(2 \partial_{z z}^{2} w+\partial_{x x}^{2} w+\partial_{x z}^{2} u\right)-\partial_{z \prime} \tau_{z z} \cos \theta=0$.

where $p$ is hydrostatic pressure, $\mathbf{v}=(u, w)$ is the velocity field in the amorphous layer, and $\tau$ is a stress tensor comprising the cumulative effect of the damage produced by irradiation, $z^{\prime}$ being a coordinate along the ion-beam direction. In Eqs. (2) and (3) ion damage occurs through the space variation of $\tau$, which depends on the distance to the surface. It is difficult to obtain analytical results for the functional form of $\partial_{z^{\prime}} \tau_{z z}$ from e.g. MD data [4]. However, we can approximate this term as a finite difference between the amorphouscrystalline $(a-c), h_{a c}$, and the free, $h$, interfaces, as

$\partial_{z^{\prime}} \tau_{z z} \simeq \frac{\tau_{z z}(h)-\tau_{z z}\left(h_{a c}\right)}{d_{z^{\prime}}} \equiv \frac{\Delta \tau_{z z}}{d_{z^{\prime}}}$,

where $d_{z^{\prime}} \approx R_{0} / \cos \left(\gamma_{0}\right)$ is the distance between both interfaces along $z^{\prime}$, and $R_{0}$ is the average layer thickness, $\gamma_{0}$ being the local incidence angle [4].

The boundary conditions at the free interface $z=h(x, t)$ implement both surface tension and the effect of the ion-induced stress $[2,3,14]$. Moreover, the dynamics of the height fields $h(x, t)$ and $h_{a c}(x, t)$ are dictated by the fluid motion through

$$
\begin{aligned}
& \left.\frac{D z}{D t}\right|_{z=h}=w(z=h)+j_{e r}, \\
& \left.\frac{D z}{D t}\right|_{z=h_{a c}}=w\left(z=h_{a c}\right)+j_{a m},
\end{aligned}
$$

where $j_{e r}$ and $j_{a m}$ account for the rates of erosion and amorphization at the free and $a-c$ interfaces, respectively. Setting $j_{e r}=j_{a m}$ guarantees a stationarity density for the layer. Additionally, the tangent component of the fluid velocity is set to zero (no slip) at $z=h_{a c}$.

Using the standard theory of pattern formation [15], the information at the early stages of the dynamics can be extracted from the linear dispersion relation $\omega_{q}$ characterizing periodic perturbations of a flat solution,

$$
\begin{aligned}
h(x, t) & =\epsilon h_{1} \mathrm{e}^{\omega_{q} t+i q x}, \\
h_{a c}(x, t) & =-R_{0}+h\left(x-R_{0} \tan \theta, t\right) \\
& =-R_{0}+\epsilon h_{1} \mathrm{e}^{\omega_{q} t+i q\left(x-R_{0} \tan \theta\right)} .
\end{aligned}
$$

Note that, as observed in the experiments [4], the $a-c$ interface is vertically and horizontally displaced by $-R_{0}$ and $-R_{0} \tan \theta$, respectively, with respect to $h$. A more elaborate relation can be written; however, Eq. (8) suffices up to the present order of approximation.

Near the onset of ripple formation, e.g. at $\theta \gtrsim 45^{\circ}$ for $\mathrm{Si}$, it is believed that purely erosive effects can be neglected [1]. In view of this fact and for simplicity, we set $j_{e r}=j_{a m}=0$. As indicated, the linear dispersion relation can be obtained from the equations above, its imaginary part controlling the pattern propagation for the unstable modes. Since the ripple wavelength is typically much larger than the thickness of the amorphous layer, namely $R_{0} q \ll 1$, we can expand $\omega_{q}$ in powers of $q$. Its imaginary part yields

$$
\begin{aligned}
\operatorname{Im}\left(\omega_{q}\right)= & \frac{-3\left[\Delta \tau_{z z}+2 \tau_{z z}(0)\right]+\cos (2 \theta)\left[5 \Delta \tau_{z z}+12 \tau_{z z}(0)\right]}{6 \eta \tan \theta} R_{0} q \\
& +\frac{\sigma R_{0}^{2}}{3 \eta \tan \theta} q^{3}+\mathcal{O}\left(q^{5}\right) .
\end{aligned}
$$

\subsection{Patterns and ripple velocity}

The existence or absence of pattern is controlled by the real part of $\omega_{q}$, as discussed elsewhere [2,4]. E.g., the non-trivial form of $h_{a c}$ in Eq. (8) is seen to lead to values of $\theta_{c}$ which depend, as in experiments, on the ion/target combination [4]. This contrasts with previous predictions on an universal $\theta_{c}=45^{\circ}$ value [14].

Likewise, the velocity at which the linear ripple structure travels across the substrate equals the phase velocity of a wave mode, being given by [16]

$$
\begin{aligned}
V_{\text {stress }} & =-\frac{\operatorname{Im}\left(\omega_{q_{m}}\right)}{q_{m}} \\
& =V_{c} S_{a c}[-99+339 g+32(4-13 g) \cos (2 \theta) \\
& +(3-51 g) \cos (4 \theta)] / \tan (\theta),
\end{aligned}
$$

where $q_{m}$ is the wave-number at which $\operatorname{Re}\left(\omega_{q}\right)$ takes its maximum positive value. Positive velocity values indicate that ripples propagate in the same direction as the ion projection on the surface. In Eq. (10) the dimensionless parameter $g \equiv \tau_{z z}(0) / \tau_{z z}\left(h_{\mathrm{ac}}\right)$ describes the inhomogeneity of the ion-induced stress distribution, $V_{c} \equiv R_{0} \tau_{z z}\left(h_{\mathrm{ac}}\right) / 192 \eta$ is a characteristic velocity scale in the problem, and $S_{a c} \equiv \operatorname{sign}\left(\tau_{z z}\left(h_{a c}\right)\right)$.

We next include the effects of sputtering within the simplest approximation: similarly to the classic BH derivation [11], to linear order in height derivatives one can simply add the corresponding prediction of Sigmund's theory [17], where the ripple velocity is given by $[9,18]$

$V_{\text {sputtering }}=\frac{J}{n}\left(Y(\theta) \sin \theta-\frac{d Y(\theta)}{d \theta} \cos \theta\right)$,

where $J$ is the ion flux, $n$ is the surface atomic density, and the sputtering yield $Y(\theta)$ is given by,

$$
\begin{aligned}
Y(\theta)= & Y(0) \exp \left[-\frac{a^{2} / \sigma^{2}}{2\left(1+b^{2} \mu^{2} / \sigma^{2}\right)}+a^{2} / 2 \sigma^{2}-\Sigma \sqrt{1+b^{2}}\right] \\
& \times \sqrt{\frac{1+b^{2}}{1+b^{2} \mu^{2} / \sigma^{2}}},
\end{aligned}
$$

where $b=\tan \theta, \Sigma$ is the Yamamura coefficient and $a, \mu$, and $\sigma$ parameterize Sigmund's Gaussian distribution [18]. Thus, the total ripple velocity is the sum of Eqs. (10) and (11). The relative weights of each contribution will depend on $V_{c}$ and $J / n$. For the sake of clarity, we introduce a new dimensionless parameter,

$B \equiv \frac{V_{c}}{J / n}=\frac{R_{0} \tau_{z z}\left(h_{\mathrm{ac}}\right) n}{192 J \eta}$. 


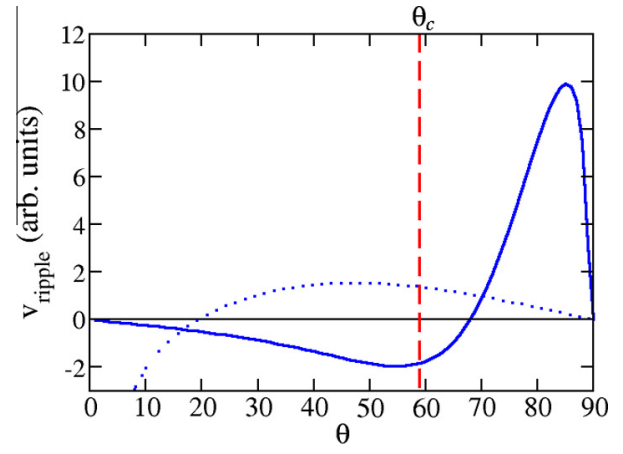

Fig. 1. Comparison between the velocity of the ripple due to stress for $g=-0.5$ (dotted blue line) and the prediction from sputtering alone (solid blue line). The vertical red dashed line shows the critical angle $\theta_{c}$ above which the patterns can be observed (the surface is flat below that value). (For interpretation of the references to color in this figure legend, the reader is referred to the web version of this article.)
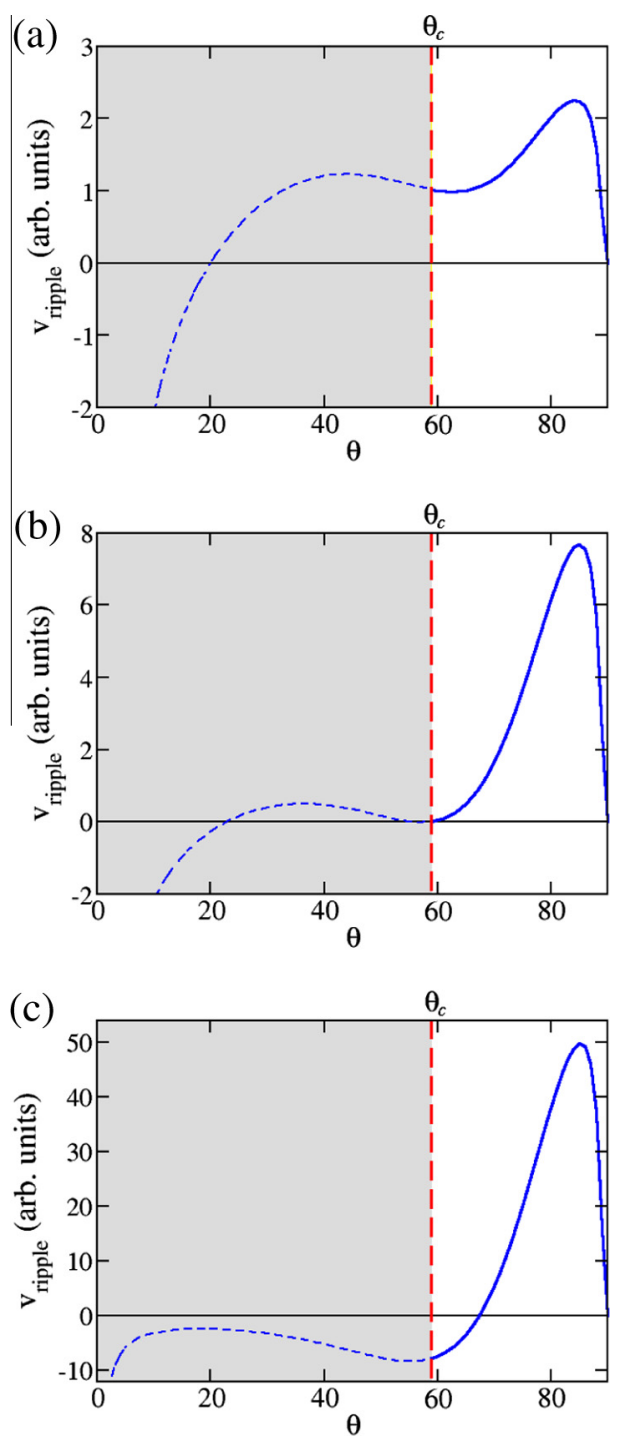

Fig. 2. Ripple velocity under a non-BH type scenario $g=-0.5$ (as in Ref. [4]) for $B=5(\mathrm{a}), B=0.75$ (b), and $B=0.2$ (c). In each panel the total ripple velocity $V_{\text {stress }}+V_{\text {sputtering }}$ is plotted vs incidence angle $\theta$. The dashed vertical line indicates the value of the critical angle $\theta_{c}$ above which ripples form. Dashed blue lines and shaded areas correspond to regions where there are no ripples. Positive values indicate that ripples propagate in the direction of the ion projection. (For interpretation of the references to color in this figure legend, the reader is referred to the web version of this article.)
When $B \ll 1(B \gg 1)$, only the effects of sputtering (stress) are taken into account. As an illustration, in Fig. 1 we compare the velocity of the ripples according to our theory with $g=-0.5$, Eq. (10), and erosive contributions coming from Eq. (11).

\section{Results}

As discussed in Ref. [4], the sign of the real part $\operatorname{Re}\left(\omega_{q}\right)$ controls the value of the critical angle, $\theta_{c}$, that separates two regions in the $\theta$ axis corresponding to different morphologies, either rippled of flat (shaded areas in Fig. 2). This sign depends on the distribution of ion-induced stress, which is primarily determined by the ion/target combination. As mentioned in Section 1, two scenarios are found in [4]: (i) non-BH type scenario for $g<1 / 4$, which is the one usually observed in semiconductor materials, where ripples only form for angles $\theta>\theta_{c}$; (ii) $\mathrm{BH}$ type scenario for $g>1 / 4$, in which parallel mode ripples occur only for $\theta<\theta_{c}^{*}$. We discuss both scenarios separately.

\subsection{Non-Bradley-Harper type scenario}

In Fig. 2 we show the predictions of the ion-induced solid flow theory in combination with the Sigmund-Yamamura prediction for the ripple velocity, for different relative weights (through the parameter $B$ ) between both mechanisms. When the ripple velocity is dominated by stress, Fig. 2a, the theory predicts ripple propulsion (namely, ripple motion is parallel to the projection of ion incidence) for all incidence angles above a new critical value, $\hat{\theta}_{c}$. This value is independent of $\theta_{c}$ and is located around $20^{\circ}$, hence outside the $\theta$-region in which ripples actually form. Moreover, $\hat{\theta}_{c}$ is robust with respect of the values of $g$ while, as shown in Ref. [4], $\theta_{c}$ depends strongly on the distribution of ion-induced stress, perhaps as in experiments. In Fig. $2 \mathrm{c}$ we show the case where sputtering is the dominant mechanism behind ripple propagation. In this case, using the parameters in Ref. [6,7] we obtain a transition in the sign of the ripple velocity around $65-70^{\circ}$, akin to recent experiments [10].

\subsection{Bradley-Harper type scenario}

In this case, and depending on the specific values of $g$ and $B$, the situation is more peculiar. For instance, for $g=1$ the patterns would be observable for $\theta<\theta_{c}^{*}=65^{\circ}$ and the ripple velocity would be as in Fig. 3. Again, depending on the values of $g$ and $B$, there can

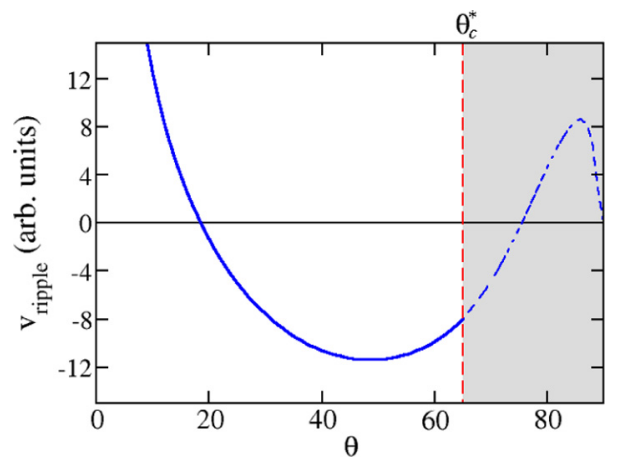

Fig. 3. Ripple velocity under a $\mathrm{BH}$ type scenario $g=1$ for $B=5$. In this case, the sign of the contribution due to stress is opposite to the one it takes in the non-BH type scenario. The dashed vertical line indicates the value of the critical angle $\theta_{c}^{*}$ below which ripples form. Dashed blue lines and shaded area correspond to the region where there are no ripples. (For interpretation of the references to color in this figure legend, the reader is referred to the web version of this article.) 
be two transitions from positive (ripple propulsion) to negative velocity and, finally, again to positive. This re-entrance could be tuned to fit into the range of angles where the pattern can be observed (not shown).

\section{Discussion and conclusions}

We have presented a qualitative account of the relevance of the competition between stress and erosive effects in the propagation of ripples. While it had been previously shown [4] that stress explains the lack of patterns below/above a certain critical angle $\theta_{c}$ that depends on the state of stress, here we have shown that erosive mechanisms (that emphasize the role of surface effects) need to be weighted with respect to stress in order to account for transitions in the sign of the ripple velocity.

Further improvements of the work presented here will be considered along two alternative pathways. On the one hand, a more detailed theory that includes all the effects of erosion (through the erosion and amorphization currents $j_{e r}$ and $j_{a m}$ ) is required to produce more quantitative predictions. On the other hand, the simple heuristic description of the amorphous-crystalline interface as a translation of the free surface, through Eq. (8), can be improved using a more mechanistic physical description of the stress confined in the amorphous layer in top of a crystalline tensionless material. Finally, from a mathematical viewpoint, we want to emphasize that the shallow-water approximation might be inaccurate in some cases and, hence, a fully non-local dispersion relation [2] (or refined expansions) is mandatory to capture the complexity of the process.

\section{Acknowledgments}

We gratefully thank C. Ballesteros, R. Gago, B. Galiana, A. Redondo-Cubero, and L. Vázquez for the collaboration leading to [4]. Our work has been funded through MINECO (Spain) grants FIS2012-38866-C05-01, FIS2012-32349, and FIS2013-47949-C2-2. A.M.-B. acknowledges support from MINECO, through FPI scholarship BES-2010-036179.

\section{References}

[1] J. Muñoz-García, L. Vázquez, M. Castro, R. Gago, A. Redondo-Cubero, A Moreno-Barrado, R. Cuerno, Mater. Sci. Eng. R 86 (2014) 1.

[2] M. Castro, R. Cuerno, Appl. Surf. Sci. 258 (2012) 4171.

[3] M. Castro, R. Gago, L. Vázquez, J. Muñoz-García, R. Cuerno, Phys. Rev. B 86 (2012) 214107

[4] A. Moreno-Barrado, M. Castro, R. Gago, L. Vázquez, J. Muñoz-García, A. Redondo-Cubero, B. Galiana, C. Ballesteros, R. Cuerno, Phys. Rev. B 91 (2015) 155303.

[5] H. Hofsäss, Appl. Phys. A 114 (2014) 401

[6] C.S. Madi, B. Davidovitch, H.B. George, S.A. Norris, M.P. Brenner, M.J. Aziz, Phys. Rev. Lett. 101 (2008) 246102.

[7] C.S. Madi, H.B. George, M.J. Aziz, J. Phys.: Condens. Matter 21 (2009) 224010.

[8] M. Engler, S. Macko, F. Frost, T. Michely, Phys. Rev. B 89 (2014) 245412.

[9] P.F.A. Alkemade, Phys. Rev. Lett. 96 (2006) 107602.

[10] H. Hofsäss, K. Zhang, H. Gehrke, C. Brüsewitz, Phys. Rev. B 88 (2013) 075426.

[11] R.M. Bradley, J.M.E. Harper, J. Vac. Sci. Technol. A 6 (1988) 2390.

[12] R.M. Bradley, P.D. Shipman, Phys. Rev. Lett. 105 (2010) 145501.

[13] R.M. Bradley, Phys. Rev. B 87 (2013) 205408.

[14] S.A. Norris, Phys. Rev. B 86 (2012) 235405.

[15] M. Cross, H. Greenside, Pattern Formation and Dynamics in Nonequilibrium Systems, Cambridge University Press, Cambridge, England, 2009.

[16] R.M.M. Mattheij, S.W. Rienstra, J.H.P. ten Thije Boonkkamp, Partial Differential Equations: Modeling, Analysis, Computation, SIAM, Philadelphia, 2005.

[17] P. Sigmund, Phys. Rev. 184 (1969) 383.

[18] B. Davidovitch, M.J. Aziz, M.P. Brenner, Phys. Rev. B 76 (2007) 205420. 